Review Article in Technology

\title{
The Relationship between AI and Humans in the Future
}

\author{
Yide Song ${ }^{1, *}$ \\ ${ }^{1}$ Northeastern University, Boston, Massachusetts 02115, USA \\ *Correspondence: s877267524@outlook.com
}

(Received: 08/06/2020; Accepted: 09/07/2020; Published: 10/09/2020)

DOI: https://doi.org/10.37906/isteamc.2020.8

\begin{abstract}
The relationship between human and Artificial intelligence (AI) is an arguable topic. In some fictions, AI has been considered as a potential risk for human. However, this topic has seldom considered from both technology and fictions. In this paper review the recent AI technology and fictions. This article presents the hope of AI in daily life and science research, creation and basic algorithm. It shows that people will rely more on AI, however it is impossible to replace human without breakthrough in future development. In the end, this paper justified that AI need restriction.
\end{abstract}

Keywords: AI; relationship between human and AI; technology

\section{Introduction}

In Stanley Kubrick's 2001: Space Odyssey (1968), “Hal”, an artificial intelligence, makes it possible to fly to Jupiter. It works like a human astronaut, and even better, it won't make any mistakes. At first, it cooperates with crew members well, but in the middle of the trip, "Hal" takes over the ship and intentionally tries to kill all crew members. All crew members are murdered except Dave. Is AI a real threat to humans as it's predicted in Kubrick's 2001? Will AI replace humans as in the Terminate movies? This paper will explore these questions.

\section{Analysis}

2.1 AI impacts human's future daily life and science research

People will indeed rely more on AI in the near future as some movies depict. Some of the predictions have become reality. For example, Robonaut 2, a robot astronaut is a crew member for International Space Station (ISS) like "Hal" in "Space Odyssey". It is a helper for the human crew. In the future, AI will play an important role in people's daily life. With the development of AI and the Internet of things (IoT), "J.A.R.V.I.S" in "Iron man" is likely to be achieved in the coming years. Jack Ma and Elon Musk mention that people will have fewer work hours and fewer jobs than now. One reason is that AI has better performance than humans in long working hours because people will make mistakes due to tiredness, but AI doesn't have that problem. So, for a long-distance trip, self-driving AI is much safer than a human driver because it will make fewer mistakes than humans. Beyond daily use, AI technology will also help people better discover the world. "Neuroscience plays a key role in the history of AI". It gives people the basic idea of AI structure. At the same time, "draw inspiration in AI research is important". Intel uses AI to develop an analysis kit for brain image in FMRI (functional magnetic resonance imaging), which is a research method especially made for the brain. It can help scientists understand how people's brains work. 
DeepMind scientists also mention that "the quest to develop AI will ultimately also lead to a better understanding of our minds". Now, AI is employed in more areas. It is inevitable for people to use this technology in the future.

\subsection{Relationship between AI creation and human}

Although AI will play an important role in people's lives, it is still not able to replace humans. AI will not be a threat to humans as predictions in science fiction for several reasons. At the moment, AI needs humans to build its structure. In many films about AI, they always choose to be blind to the process of building AI. For example, in Alex Garland's "Ex Machina" (2015), it focuses on AI testing instead of building. That is the reason why AI can build themselves in fiction. However, in reality, it is hard to achieve. Just as humans acquire knowledge from textbooks, AI has a similar learning process: it "studies" its "textbook" -- a large "training set", which includes the features that people hope AI can recognize. OpenAI, a world-famous AI research company, mentions "think millions of images" on their website. Now, some AI may not need such a "training set". Take DeepMind's "AlphaGo Zero" as an example, it uses a different learning method called reinforcement learning (RL) which can learn from their mistakes. Unfortunately, this method is not suitable for all situations. Even if AI does not need a "training set", humans are still actively involved in the second process---building "neural networks".

Only human programmers can create the "neural network", which is the framework for AI. It actually is a mathematical model. Therefore, it just uses math methods to simulate how the brain works in different situations. Neural networks have different benefits and limits, so networks are diverse in AI due to their jobs. For instance, the "convolutional neural network" (CNN) has succeeded in "image recognition", but text classification needs "recurrent neural network" (RNN). It is not an easy job to create a neural network especially for these hard tasks like playing Go. Therefore, some companies develop a tool called "Auto ML" to "build" a neural network. It is always considered as "creator". In fact, it is just a selection algorithm which could only select something that existed instead of building. Also, this technology is only suitable for some tasks which already have a lot of data and models.

After an AI is successfully built, just like other programs, it needs to be developed. This part still needs humans, especially users' help: just like students will take exams to test whether they have problems with some topics. Although AI has already been tested several times in the training process, the data selected are just a sample that can't cover everything. That is the reason why users' feedback is important for AI, especially virtual assistants like Siri. In Apple's privacy policy, it mentioned that "You can opt in to have the audio of your interactions with Siri and Dictation stored and reviewed by Apple employees to develop and improve Siri". It implies that Apple is collecting data for another round of training for Siri so that it can answer more questions and fix more problems. With more data, the assistant can answer more questions and fix some problems. Other companies also apply the same process. Hence, it is impossible for AI to develop by themselves. In the future, fewer people need to work on creating an AI. However, people will keep approaching the goal of self-development AI, which can't be achieved unless a technology revolution occurs.

\subsection{The intelligence of AI}

In many fictions, people are worried that AI will become "self-recognized" or "self-conscious", which means AI will "be aware of itself" and "use mental power to understand what happens". Take Jonathan Nolan's "Westworld" as an example, "hosts" have independent thinking in this series. They realize that they are trapped in the Westworld. To get freedom, they start a revolution to fight against humans. Audiences are concerned that it will happen in the future. But in reality, AI is far from achieving selfconsciousness, and the revolution, should it come, will happen only in a distant future. 
Intelligence is a basic requirement for self-recognition. Although some AI may pass the Turing test now (The result is controversial), it is still arguable that AI has intelligence. Like Jake may say, "computer is computer". AI's intelligence is different from human intelligence. Unlike humans' problems solving process, which involves comprehension, AI gets answers to questions purely based on mathematics. For example, a sample AI system distinguishes different objects. The algorithm for it is using some method and the "training set" to approach a suitable function. Assuming the function in a coordinate is like Figure 1. Then, if two features (Feature 1 and 2) of one object that are measured in these two axes can create a point in section $A$, it is Object type X. If this point is in section B, it is object type Y. After several rounds of training, the function will approach the accurate function. So, it is hard to determine whether AI has intelligence or not.

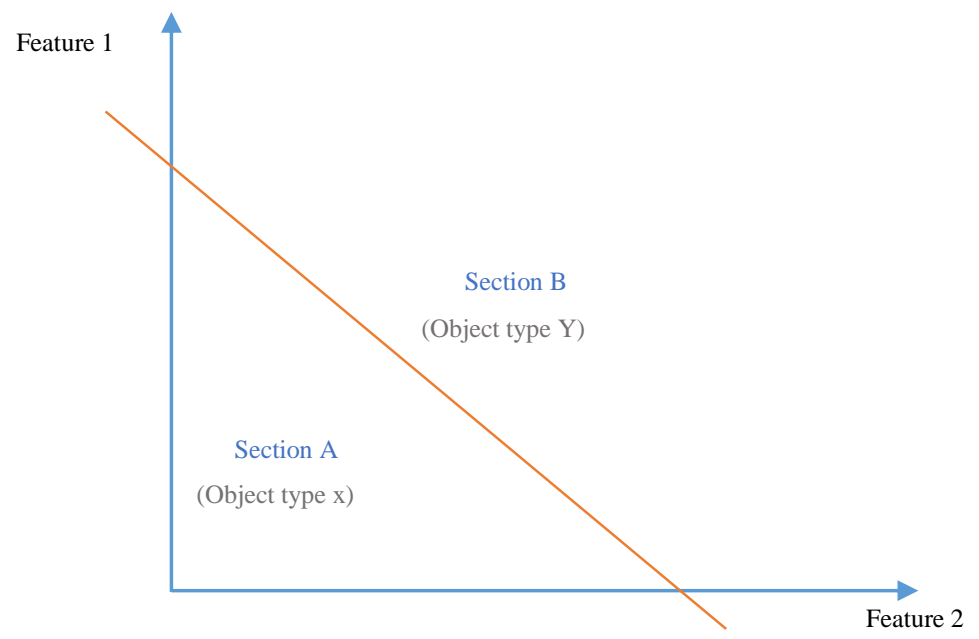

Figure 1. A simple feature space with linear classifier

Even when this type of "intelligence" is considered as real intelligence, a huge gap still exists between the mathematical method and "self- recognize". One important term for "self-recognized" is "understanding". AI can master many things after training, like AlphaGo can defeat a human champion in Go. However, it is hard to say that it understands Go very well. AlphaGo is similar to others using a mathematical method to know where the next move should be placed. It will calculate the possibility of winning before the move. Then, it will place the Go stone to where has the highest winning possibility. Similar to people who take multiple-choice questions, AlphaGo starts to calculate the possibility of correct for each choice. It may be hard to believe, but this process doesn't require AI to understand the question itself. It can be explained by "Chinese room Argument". The experiment is about a man who doesn't know any Chinese language gets locked in a room and asked to answer questions in Chinese. He has boxes filled with Chinese symbols and instructions for answering questions with these symbols. When people send Chinese questions to him, he just uses the instructions to get the correct answer. But he still doesn't understand any Chinese. So, for AlphaGo and other AI, it is highly possible that they just know the correct answer. Because after a million rounds of testing, AI could approach a perfect function. Then it just uses this function to approach the answer. But in fact, they may don't understand why the answer should be that. It is like a person who guesses every multiple-choice question. Although he doesn't know the process of solving problems, his guess is always correct. 


\section{Conclusions}

In the future, it will still be hard for AI to have consciousness because it relates to basic AI knowledge. To make a breakthrough, people need to have a deeper understanding of that. It requires a breakthrough in neuroscience and psychology. It is impossible to stop AI technology's development. Although people should not consider AI a threat, people do need to worry about the lack of policies to regulate the usage of it. For example, Deepfake can replace one person in a video with others' likeness. This technology doesn't require a lot of knowledge about AI. At first, it causes some rumors spreading in society due to lack of restrictions. These fake videos have a negative impact on some public figures. Forbes describes it as "going to wreak Havoc on society". Fortunately, Deepfake regulations are announced by some governments and legislatures now. But if there are no restrictions to AI, worse things will occur in future.

\section{References:}

AI in Neuroscience - Helping Better Understand the Brain. (n.d.). Retrieved August 24, 2020, from http://www.intel.com/content/www/us/en/analytics/artificial-intelligence/ai-in-neurosciencevideo.html.

Ask Siri, Dictation \& Privacy. (2019, October 28). Retrieved August 24, 2020, from https://support.apple.com/en-us/HT210657

AutoML. (n.d.). Retrieved August 24, 2020, from http://www.automl.org/automl/

Cloud Auto ML - Custom Machine Learning Models I Google Cloud. (n.d.). Retrieved August 24, 2020, from https://cloud.google.com/automl/

Dunbar, B. (2010, March 02). Robonaut2, the Next Generation Dexterous Robot. Retrieved August 24, 2020, from http://www.nasa.gov/topics/technology/features/robonaut_photos.html

Elon Musk and Jack Ma disagree about AI's threat. (2019, August 29). Retrieved August 24, 2020, from http://www.bbc.com/news/technology-49508091

Hassabis, D., Kumaran, D., Summerfield, C., \& Botvinick, M. (2017). Neuroscience-Inspired Artificial Intelligence. Retrieved August 24, 2020, from www.cell.com/neuron/fulltext/S0896-6273(17)305093?_returnURL=https\%3A\%2F\%2Flinkinghub.elsevier.com\%2Fretrieve\%2Fpii\%2FS0896627317305 093\%3Fshowall\%3Dtrue

Hassabis, D., Summerfield, C., \& Botvinick, M. (2017, August 02). AI and Neuroscience: A virtuous circle. Retrieved August 24, 2020, from https://deepmind.com/blog/article/ai-and-neurosciencevirtuous-circle

Hornby, A. S., Zhao, C., \& Zou, X. (2014). Niu jin gao jie ying han shuang jie ci dian = Oxford advanced learner's English-Chinese dictionary. Beijing: Shang wu yin shu guan niu jin da xue chu ban she(zhong guo)You xian gong si.

Karpathy, A. (2019, March 08). Generative Models. Retrieved August 24, 2020, from https://openai.com/blog/generative-models/

Machine Learning Glossary I Google Developers. (2020). Retrieved August 24, 2020, from https://developers.google.com/machine-learning/glossary/

Searle, J. R. (1980). MINDS, BRAINS, AND PROGRAMS. Retrieved August 24, 2020, from http://cogprints.org/7150/1/10.1.1.83.5248.pdf 